\title{
Competitividad urbana en México: una propuesta de medición.*
}

\author{
ENRIQUE CABRERO MENDOZA**, ISELA ORIHUELA JURADO***, ALICIA ZIC- \\ CARDI CONTIGIANI**** \\ ** Centro de Investigación y Docencia Económicas, AC, México. \\ *** Centro de Investigación y Docencia Económicas, AC., México. \\ **** Programa Universitario de Estudios de la Ciudad - UNAM
}

\begin{abstract}
Urban competitiveness has been a recurrent topic during recent decades. Diverse articles have been published on this issue, showing rankings of countries or cities around the world, based on diverse factors. Nevertheless, these studies do not reveal the particularities of the cities in each country and they lead to unclear interpretations of results. This article presents a methodological proposal to build a competitiveness index for Mexican cities -2003 and 2007-derived from a literature review and a resulting model based on four components: urban-environmental, economic, socio-demographic and institutional. The objective is to show the characteristics and capacities of the cities of a country in terms of competitiveness and to provide useful elements for public policy formulation.
\end{abstract}

KEY WORDS: metropolitan areas, urban competitiveness.

RESUMEN La competitividad urbana constituye un eje de análisis recurrente en las últimas décadas. Diversos artículos que se han publicado sobre este tema muestran rankings de países o ciudades a lo largo del mundo, basados en factores particulares. Sin embargo, esta clase de trabajos no muestran las particularidades de las ciudades de cada país y llevan a una interpretación poco clara de los resultados. En este artículo, en cambio, se presenta una propuesta metodológica para construir un índice de competitividad para las ciudades mexicanas -2003 y 2007- basada en la revisión de la bibliografía y en la elaboración de un modelo a partir de cuatro componentes: urbano-ambiental, económico, socio-demográfico e institucional. El objetivo es mostrar las características y capacidades de las ciudades en un país en términos de competitividad y provisión de elementos útiles para la formulación de políticas públicas.

PALABRAS CLAVE: áreas metropolitanas, competitividad urbana.

\footnotetext{
* Recibido el 23 de octubre de 2008, aprobado el 25 de enero de 2009.

Correspondencia: Enrique Cabrero Mendoza, Carretera México-Toluca 3655, Col. Lomas de Santa Fe, CP. 01210, México, DF. E-mail: enrique.cabrero@cide.edu. Isela Orihuela Jurado, Carretera México-Toluca 3655, Col. Lomas de Santa Fe, CP. 01210, México DF. E-mail: isela.orihuela@cide.edu. Alicia Ziccardi Contigiani, Moneda n 2, Centro Histórico, C.P. 06060. México D.F. E-mail: ziccardi@servidor.unam.com
} 


\section{Introducción}

A nivel mundial las ciudades juegan actualmente un papel fundamental como motor del desarrollo económico, en tanto son los espacios de concentración de nuevas tecnologías e infraestructuras de la información y la comunicación, los servicios especializados, los centros de ciencia y tecnología, así como de personal de la alta calificación; factores estos entre otros que atraen y retienen la inversión de capital, generando empleo. La creación de estas condiciones que ofrecen las ciudades ha dado origen a numerosos trabajos sobre la llamada competitividad urbana, un término bastante impreciso y polémico, que para algunos autores como Porter (1995, 1996), Lever y Turok (1999), Begg (2002), Moori-Koening y Yoguel (1998) y Sobrino (2002) alude a un proceso de generación y difusión de competencias, donde no sólo los factores micro-económicos tienen relevancia, sino también las características que ofrece el territorio para facilitar las actividades económicas. Las ciudades, entonces, pueden ser generadoras de un entorno físico, social, económico e institucional capaz de incentivar la inversión privada y apoyar el desarrollo de actividades productivas.

En el marco de estas preocupaciones, el principal objetivo de este artículo es presentar los resultados de un esfuerzo metodológico realizado para medir las condiciones de competitividad urbana que ofrecen las zonas metropolitanas del México actual, combinando diferentes componentes: institucional, urbano-ambiental, económico y socio-demográfico. La finalidad es construir perfiles de ciudades y ofrecer lineamientos generales para orientar las políticas públicas desde el ámbito del gobierno local.

\section{Sobre la competitividad urbana}

La competitividad ha sido utilizada como un elemento para evaluar la participación de las ciudades en el ámbito nacional e internacional. Además, representa una herramienta de política urbana para hacerlas más atractivas a la inversión por medio de mejoras en la infraestructura para el desarrollo económico y en los servicios que brinda a la población. Gordon (1999) menciona que la competitividad se ha convertido en un medio para explorar estrategias que resuelvan los problemas urbanos. Es por ello que el papel de los actores gubernamentales, sociales y privados cobra relevancia en este contexto, ya que a través de sus asociaciones o redes pueden funcionar como agentes promotores de la inversión, servicios avanzados, mano de obra calificada, obtener fondos públicos, propiciar el desarrollo de sectores de alta tecnología, turismo, eventos internacionales $y$, por ende, brindar mejores condiciones de vida para los habitantes de las ciudades.

Así, la competitividad se convierte en un factor determinante en el desarrollo urbano y regional, ya que a través de acciones y políticas implementadas por los gobiernos locales se puede lograr que estos espacios participen en el mercado nacional, regional e internacional de bienes y servicios, incrementen su ingreso real y el bienestar de sus ciudadanos, y promuevan un desarrollo sustentable. Por lo general, es una acción público-privada, dado que las ciudades o las regiones son un sujeto colectivo conformado por autoridades locales, empresarios, y organizaciones económicas y sociales que pueden ser promotoras de su propio desarrollo económico. 
Es claro que una ciudad competitiva no es aquélla que sólo logra captar inversiones por sus bajos costos de mano de obra, sino aquélla capaz de crear mejores condiciones, capacitando más a la fuerza de trabajo, elevando la productividad y ofertando mejores condiciones urbanoambientales e institucionales, ya sea en términos de infraestructura básica y de comunicaciones, como en calidad de los servicios especializados, innovaciones tecnológicas, medio ambiente sustentable, instituciones transparentes, calidad en las regulaciones, combate a la inseguridad y promoción de la cohesión social; es decir, mejores condiciones generales para la producción económica y para el desarrollo de la vida social. La competitividad, por tanto, es un conjunto de elementos en los que incluso el tema del abatimiento de las inequidades sociales se convierte en una palanca fundamental para la atracción de inversión y oportunidades ${ }^{1}$.

Pero no todas las ciudades compiten bajo los mismos parámetros. Porter (1995) ha afirmado que la competitividad es un proceso altamente localizado que promueve la especialización y la eficacia local. Por lo tanto, cada lugar ofrece ventajas específicas dependiendo de sus diversos factores de atracción, a lo que se le llama "ventajas competitivas". Este autor identifica dos tipos: las estáticas y las dinámicas. Las primeras son el resultado de la aglomeración o concentración geográfica y de la desintegración vertical. Entre ellas se encuentran la localización geográfica, disponibilidad de infraestructura y estándares medioambientales. Las segundas surgen de la capacidad innovadora y son creadas por los gobiernos, empresas, asociaciones y/o redes de ciudades. Se pueden mencionar en este grupo a las universidades y centros de investigación, personal calificado, gobiernos locales promotores de la economía local y capacidad de innovación tecnológica y empresarial.

\section{Factores que definen a la competitividad urbana (ventajas competitivas)}

Las condiciones cambiantes producidas por el proceso de globalización provocan que las ciudades se mantengan en una constante adaptación para crear, mantener o mejorar sus condiciones de participación dentro del mercado global y ser así más competitivas. El grado de participación depende de factores sociales, económicos y políticos de cada lugar (Raco, 1999).

Existen diversos enfoques para identificar los elementos que definen la competitividad urbana. Uno de ellos los divide en determinantes económicos y estratégicos (Kresl, 1995). Los económicos se refieren a cuestiones cuantitativas como los factores de producción, infraestructura, localización, estructura económica y amenidades; mientras que los estratégicos son elementos cualitativos ligados a la política pública, estrategia urbana, cooperación entre los sectores público y privado y diseño institucional.

Asimismo, la competitividad puede definirse al tratar de interrelacionar los factores que afectan el desempeño económico urbano. Para ello, Begg (1999) menciona que la tasa de empleo, productividad y niveles de vida son los que determinan el desempeño de las ciudades y, por lo tanto, su nivel de competitividad. Sin embargo, estos se ven influenciados por

$1 \quad \mathrm{Al}$ respecto, se han desarrollado interesantes aportes de estudios que muestran evidencia de la fuerte correlación entre un alto nivel de competitividad y un bajo nivel de desigualdad social, así como la fuerte propensión a que ambos procesos vayan aparejados. Véase Pastor (2006). 
el comportamiento sectorial, es decir, el tipo y funciones de las empresas, por los factores de atracción para invertir en el lugar, y por la capacidad de las empresas para desarrollar nuevos procesos y productos. En este sentido, la capacidad que tiene una ciudad para competir depende tanto de sus atributos locacionales como de las debilidades y fortalezas de las empresas y demás agentes económicos que participan en ella.

La competitividad también puede definirse por la forma en que encaja un lugar en el sistema urbano a través de cuatro aspectos principales: carácter jerárquico, patrón de especialización/ diversificación de actividades y productos, división funcional del trabajo, y papel de las relaciones interurbanas (Gordon, 1999). Tanto la estructura económica, el lugar en el sistema urbano, las características del empleo y las redes que se tengan establecidas entre las ciudades, son aspectos que definen la capacidad de las ciudades para ser competitivas.

Lever (1999) menciona que debido a que los objetivos de la competencia entre ciudades son la inversión, turismo, población, fondos públicos y eventos de alto nivel, el éxito de una ciudad depende de la existencia y manejo de factores distributivos, desarrollo económico, sustentabilidad y calidad de vida. La calidad de vida, el medio ambiente, el nivel de servicios culturales y el acceso al conocimiento son factores importantes para la localización de personas y negocios dentro de un contexto competitivo, pues funcionan como atractivos para el capital e influyen en los patrones de crecimiento y desarrollo urbano. Rogerson (1999) y Berg y Braun (1999) mencionan que entre los factores que definen la calidad de vida se encuentran: el ambiente físico, clima, contaminación, crimen, y servicios sociales como salud y educación. También coinciden en que todos ellos son promotores de una imagen distintiva, lo cual forma una atmósfera propia para la inversión y atracción de personas.

Por otro lado, la infraestructura de la información es también un elemento importante de las ciudades globales y las redes urbanas. Las actividades, funciones y dinámica urbana dependen en gran medida de los atributos y facilidades que brindan las telecomunicaciones para apoyar el complejo de relaciones, vínculos e interacciones dentro y entre las ciudades. Sin duda, son un elemento que contribuye a su competitividad económica (Graham, 1999). El papel de las relaciones a través de personas o por medio de la tecnología, entre los sectores público y privado, forma parte importante de los elementos a considerar para la competitividad urbana. Malecki (2002) les ha llamado redes suaves y duras, y funcionan en todos los niveles, desde el global hasta el local, como insumos de información y bases para la toma de decisiones.

Las instituciones son otro factor importante en el éxito de la economía, ya que funcionan como mediadoras en las relaciones entre actores sociales y económicos-que comparten normas y convencionalismos-, facilitan nuevas formas de desarrollo económico, generan dinamismo en la utilización y generación de conocimiento y pueden contribuir a la cooperación entre los actores (Raco, 1999). Para que un gobierno funcione adecuadamente, es necesario que tenga capacidad de organización y ello incluye: organización administrativa, redes estratégicas entre los actores públicos, públicos y privados, y entre los privados; liderazgo, visión y estrategia, condiciones socio-espaciales, apoyo político, y apoyo social (Berg and Braun, 1999). 
Sobrino (2002), por su parte, propone dividir los diversos determinantes de la competitividad en tres tipos: empresariales, territoriales y distributivas. Las empresariales se refieren al comportamiento y características de las unidades productivas de la ciudad. Las territoriales se refieren a las condiciones físicas que ofrece la ciudad para desarrollar las actividades económicas. Y las distributivas se refieren a las facilidades que brinda la ciudad para la distribución y circulación de productos.

Después de haber analizado los diversos trabajos que se han escrito sobre los factores o condicionantes de la competitividad urbana, se puede identificar que en varios de ellos predominan los aspectos económicos de las zonas urbanas para determinar su grado de competitividad. Sin embargo, como se vio en el apartado anterior, el término "competitividad" no sólo alude a la parte economía urbana, sino que depende de la interrelación de diversos factores. Por ello, este trabajo tiene la intención de construir un modelo que incluye un conjunto de variables que muestran una visión más integral de la competitividad, es decir, donde las características económicas, de la población, de las condiciones urbanas y de la participación de los gobiernos locales están representadas.

Así, se integran cuatro grupos de variables: económicas, socio-demográficas, urbanas e institucionales. En las económicas se incluyen aquellas relacionadas con las características de las empresas, la productividad, la capacidad de innovación de las mismas y la estructura económica. En las socio-demográficas están las que definen el tamaño de la población, las características del empleo y la calidad de vida. Las urbanas están conformadas por las relacionadas con la infraestructura y equipamiento urbano. Mientras que las institucionales se refieren al papel de los gobiernos locales en la promoción económica de las ciudades. Más adelante se hace alusión a cada uno de estos grupos y el modelo que se utilizó en el cálculo del índice. Mientras tanto, en la siguiente sección se exponen las diversas metodologías de medición que sirvieron de base para diseñar la que se utiliza en este trabajo.

\section{Métodos para medir la competitividad urbana}

Para construir el modelo que permitiría llegar al índice de ciudades mexicanas, en primer lugar se realizó una exhaustiva revisión de la literatura con el objetivo de contar con los mayores elementos posibles que permitieran la construcción de una metodología propia para el caso de $\mathrm{México}^{2}$. Principalmente, se identificaron tres tipos de métodos dependiendo del enfoque y de los objetivos a alcanzar:

i) Los primeros son los orientados principalmente a la atracción de inversión privada, y son utilizados por consultorías o agencias gubernamentales regionales. Entre ellos se puede mencionar "Best Cities", un ranking de áreas metropolitanas de Estados Unidos que está orientado a la inversión y los negocios. Entre las variables utilizadas se encuentran: actividad empresarial, crecimiento de empresas pequeñas, crecimiento económico y riesgo. Otro es "Best Cities in Asia", cuyo objetivo es la promoción turística de las ciudades, considerando ingreso,

2 Un análisis más amplio sobre la literatura disponible en cuanto a la medición de la competitividad urbana se encuentra en Cabrero, Orihuela y Ziccardi (2003). 
desempleo, gasto en educación, número de camas en los hospitales, calidad del medio ambiente, inflación, criminalidad, medios de comunicación y esperanza de vida. Por otro lado, Mercer Human Resource and Investment Consulting Inc. presenta varios ranking internacionales de ciudades, uno de ellos se refiere al costo de vida, donde se jerarquizan las ciudades más caras y más baratas de Europa, Norte y Sudamérica, África y Medio Oriente.

ii) El segundo tipo se refiere a los que se derivan de trabajos académicos. La competitividad urbana se mide con la finalidad de enriquecer el conocimiento sobre el tema y aplicarlo en políticas públicas. Se puede mencionar el trabajo de Chesire et al. (1986), donde realiza un ranqueo de ciudades europeas de acuerdo a su competitividad. Lever (1999) por su parte, realiza un análisis para ciudades europeas, donde busca explicar el patrón de éxito económico en ańos recientes. Kresl y Singh (1999) desarrollan un método para medir la competitividad de una economía urbana individual, tanto en forma cuantitativa como comparativa. De esta manera, ranquean las 24 zonas metropolitanas más grandes de Estados Unidos de acuerdo a su competitividad. Sobrino (2002) realiza un ejercicio para 30 ciudades de México, establece variables cuantitativas y cualitativas para medir la competitividad a través de un método estadístico y realiza un ejercicio de aplicación.

iii) El tercero se refiere a aquellos trabajos que combinan los dos anteriores, es decir, están orientados a la inversión privada, pero también tienen interés de contribuir al conocimiento. En esta parte se puede mencionar el trabajo de Suzanne McCauley (2003), el cual mide la competitividad entre los países y jerarquiza las ciudades de acuerdo a su capacidad para insertarse y competir en la economía globalizada.

Para poder construir una metodología que permita medir la competitividad urbana en México, se analizaron en profundidad los trabajos académicos. En los siguientes párrafos se hace una breve explicación de las variables e indicadores utilizados en cada uno de ellos, así como los métodos llevados a cabo y los resultados obtenidos. Esta revisión fue de gran utilidad para la construcción del modelo que se presenta en este artículo. Así, Chesire et al. (1986) compara tasas de crecimiento económico e índices relacionados con el desempleo y la migración. La medición de la competitividad se realiza en términos de un promedio de problemas, donde un resultado negativo representa éxito competitivo, mientras que uno positivo representa fracaso. El estudio comprende tres periodos de tiempo separados para analizar el comportamiento de las ciudades en cada uno de ellos. Sólo considera el éxito o fracaso económico, sin tomar en cuenta las ventajas o desventajas de la estructura económica.

Lever (1999), por su parte, realiza un análisis para ciudades europeas, donde busca explicar el patrón de éxito económico en años recientes. En primer lugar, identifica tres hipótesis: i) las ciudades capitales después de la posguerra han crecido más que las demás; ii) el potencial económico, que está en función de la centralidad geográfica, explica por qué las ciudades crecen más rápido; y iii) la existencia de recursos naturales e infraestructura para la producción dados a través del tiempo representa ventajas o desventajas en cada lugar. Las variables utilizadas son: Producto Interno bruto (PIB) per cápita, 1989; tasa de crecimiento del PIB per cápita, 197585; tasa de crecimiento del PIB per cápita, 1985-89; tasa de crecimiento del PIB esperada per cápita, 1989-95; tasa de crecimiento en el VBP en servicios, 1975-85; tasa de crecimiento en 
el VBP en servicios, 1985-89, y tasa de crecimiento esperada en VBP en servicios, 1989-95. El promedio de PIB per cápita se utilizó como medida de bienestar económico. Con las tasas de los diferentes periodos se construyó un cuadro de desempeño económico para grupos de ciudades: capitales/no capitales; norte/sur; y centro/periferia. El análisis consistió en comparar los grupos de ciudades y diferenciar su desempeño económico. La independencia económica se refiere a la autonomía económica de la ciudad sobre la economía nacional.

Kresl y Singh (1999) explican el ranqueo de 24 zonas metropolitanas de Estados Unidos con un análisis de regresión y describen cada economía urbana desde el punto de vista de sus fortalezas y debilidades, en comparación con las otras 23 economías urbanas. La medida de competitividad urbana dice que la posición competitiva de la ciudad está en función del incremento en el valor bruto de la producción industrial, el crecimiento de las ventas al menudeo y el incremento de ingresos por servicios empresariales. La competitividad urbana se explica por dos tipos de elementos: económicos y estratégicos. Los económicos se midieron con 8 variables: crecimiento en el ingreso per cápita; centros de investigación/VBP manufacturera; crecimiento en el porcentaje de empresas con más de 100 empleados; número de trabajadores con valor mayor al resultado de dividir el número de trabajadores con estudios universitarios entre el total de trabajadores en el sector privado; porcentaje de trabajadores trabajando en áreas de ingeniería, administración, investigación y gestión con respecto al total; crecimiento en el número de instituciones culturales; crecimiento en el capital de reserva del Estado; exportaciones como parte de la producción total. Los factores estratégicos son cualitativos y sólo pueden medirse a través de entrevistas y análisis de documentos oficiales.

Sobrino (2002) realiza un análisis de regresión para medir la competitividad urbana. Toma en cuenta cuatro variables: cambio en la participación absoluta de una ciudad en el VBP industrial nacional entre 1988 y 1998; cambio en el crecimiento relativo de la ciudad comparado con el crecimiento a nivel nacional 1988-1998; crecimiento absoluto del VBP de la ciudad comparado con el del país entre 1988 y 1998; y cambio en la base económica. El indicador de competitividad se construye con el promedio del rango de las cuatro variables y se le llama "competitividad ponderada". Para tratar de explicar las ventajas competitivas de cada ciudad se proponen tres divisiones: empresariales, territoriales y distributivas. La competitividad ponderada es la variable dependiente y las ventajas competitivas son las variables independientes. Estas últimas se dividieron entre estáticas y dinámicas. Las variables fueron: capital, camas de hospital, estructura económica local, producción en el sector servicios, enlaces carreteros y distancia media hacia otras ciudades. Las dinámicas fueron: el valor que mostraba el cambio en productividad parcial del trabajo, sueldos, intensidad del capital, población, alumnos en educación superior, marginación, personal ocupado en parques industriales, estructura económica local, personal en maquiladoras de exportación, préstamos bancarios per cápita, ingreso público municipal per cápita y mayor dinamismo exportador a nivel nacional. El resultado muestra que las ciudades más competitivas presentan una estructura productiva diversificada, crecimiento en sus parques industriales y mayor eficiencia del gobierno en la recaudación de ingresos. La conclusión menciona que la competitividad urbana en México se sustenta en cuestiones más dinámicas que estáticas y se considera fundamental combinar la competitividad y la calidad de vida como una de las tareas de los gobiernos locales. 
En cuanto a los métodos para obtener las medidas de competitividad, Bouinot (2002) identifica tres principales:

- Métodos de indicadores de base previamente jerarquizados. Se basa el análisis de indicadores en la opinión de un grupo de expertos. Cada individuo hace una jerarquización en orden de importancia de acuerdo a su experiencia. El resultado analiza a las unidades de estudio con base en un proceso de ponderación. Musso y Castaginio (1997) utilizaron este método para medir la competitividad de ciudades del sur de Europa. The Harris Research Centre clasifica a las ciudades europeas entrevistando a 500 empresarios.

- Método de análisis factorial. Es un método para aislar diversas dimensiones dentro de un grupo de variables relacionadas. Es decir, se trata de separar patrones comunes de variación que facilitarían el análisis de alguna situación en particular. Este método se utiliza para explorar relaciones entre variables y también para generación y prueba de hipótesis. El propósito del análisis factorial es identificar y cuantificar las dimensiones supuestas para resaltar el desempeño de un grupo de variables dentro de una diversidad de cuestiones. Este método se ha utilizado en el índice de competitividad de The Global Competitiveness Report, publicado por The World Economic Forum, también para el índice de competitividad de IMD, World Competitiveness Yearbook (WCY), y en el trabajo de Chesire et al. (1986), para medir la salud urbana.

- Método de precios edónicos. Es un método muy sofisticado que consiste en considerar el precio de un bien con base en sus diversas características. Se ha aplicado para medir la calidad de vida en las ciudades. Un ejemplo es el trabajo de Giannias (1998) para ciudades canadienses, donde se incorporan variables como la temperatura, niveles de contaminación, y criminalidad.

Tanto la selección de un método de medición como la definición de variables e indicadores depende de los objetivos del estudio, de los recursos con que se cuente, del tiempo para realizarlo, así como de la información que se encuentre disponible. Para esta investigación fue de gran utilidad conocer los trabajos recientes sobre el tema, así como los argumentos teóricos principales de la competitividad urbana. De acuerdo al objetivo del presente trabajo y para poder mostrar la representatividad de cada uno de los cuatro grupos de variables indicados anteriormente, así como la integración de los cuatro en uno solo, se decidió incorporar el método de análisis factorial. La definición del modelo se presenta a continuación.

\section{Metodología para el índice de competitividad en ciudades mexicanas}

Los criterios para seleccionar las ciudades del estudio fueron tres. Primero, se realizó una primera selección con base en aquellas ciudades que para el año 2000 tuvieran una población mayor a 50 mil habitantes. De ellas, se escogieron las que presentaran los mayores niveles de producción bruta por habitante en 1998. Finalmente, para que todos los estados del país tuvieran representatividad en el índice, se incluyeron aquellas ciudades que estuvieran más cerca de cumplir con los dos requisitos anteriores. En total se eligieron 60 ciudades, algunas de ellas 
áreas metropolitanas, las que suman un total de 225 municipios $^{3}$ Cabe mencionar que en este trabajo cada unidad de estudio es llamada ciudad, integre o no un área metropolitana.

El cálculo del índice se basa en el método de análisis factorial, en donde se obtiene información a partir de cuatro componentes: económico, socio-demográfico, urbano-ambiental e institucional. Cada componente, como se mencionó anteriormente, se construyó con variables que representan su comportamiento lo más cercano posible a la realidad, y la elección de las variables se basa en la investigación teórica realizada y sustentada en diversos trabajos previos ${ }^{4}$. Cabe mencionar que la información utilizada en este índice es en su totalidad de nivel municipal, y que en México este tipo de información es limitada en comparación con el nivel estatal y federal, por lo que las variables que figuran en cada componente se limitan a la información estadística disponible en publicaciones acreditadas existentes sobre cada tema. Las variables incluidas en cada uno de los cuatro componentes se presentan en el cuadro 1.

3 Los criterios de población y valor bruto de la producción, así como la delimitación de las áreas metropolitanas mexicanas se consideraron los trabajos de Sobrino (2002 y 2003).

4 Véanse por ejemplo: Cabrero 2002; Cabrero, Orihuela y Ziccardi, 2003; Cabrero 2005. 


\section{CUADRO 1. VARIABLES E INDICADORES POR COMPONENTE DE COMPETITIVIDAD PARA EL INNICE DE CIUDADES MEXICANAS}

\begin{tabular}{|c|c|}
\hline Componente & Variables e Indicadores \\
\hline Económico & $\begin{array}{l}\text { Producción bruta total per cápita (riqueza generada) } \\
\text {, Sueldo promedio por personal ocupado (nivel salarial) } \\
\text {, Densidad de capital (activos disponibles) } \\
\text {, Índice de especialización local en industria (estructura económica de producción) } \\
\text {, Índice de especialización local en comercio (estructura económica de producción) } \\
\text {, Índice de especialización local en servicios (estructura económica de producción) } \\
\text {, Depósitos bancarios per cápita (intensidad de la actividad financiera) } \\
\text {, Participación de sectores modernos de industria (perfil y dinamismo del desarrollo) } \\
\text {, Participación de sectores modernos de comercio (perfil y dinamismo del desarrollo) } \\
\text {, Participación de sectores modernos de servicios (perfil y dinamismo del desarrollo) }\end{array}$ \\
\hline Socio-demográfico & $\begin{array}{l}\text {, Ingreso promedio de las familias (nivel promedio de vida) } \\
\text {, Índice de marginación (nivel promedio de carencias) } \\
\text {, Población económicamente activa en el sector primario (estructura de empleo) } \\
\text {, Población económicamente activa en el sector secundario (estructura de empleo) } \\
\text {, Población económicamente activa en el sector terciario (estructura de empleo) } \\
\text {, Tasa de crecimiento poblacional en la última década (nivel de atracción / expulsión) } \\
\text {, PEA con ingresos de hasta dos salarios mínimos mensuales (nivel de pobreza) } \\
\text {, Índice de desarrollo humano (potencial humano) } \\
\text {, Asegurados permanentes al IMSS (nivel de empleo formal) } \\
\text {, Tasa de desocupación abierta (desempleo) } \\
\text {, Número de delincuentes (nivel de criminalidad) }\end{array}$ \\
\hline Urbano-ambiental & $\begin{array}{l}\text { Jerarquía poblacional (tamaño de la ciudad) } \\
\text {, Servicios públicos en la vivienda (calidad de servicios en hogares) } \\
\text {, Tiendas de autoservicio (infraestructura disponible de servicios comerciales) } \\
\text {, Sucursales bancarias (infraestructura disponible de servicios financieros) } \\
\text {, Alumnos en educación superior (infraestructura y uso de servicios educativos) } \\
\text {, Camas de hospital (infraestructura disponible de servicios hospitalarios) } \\
\text {, Denuncias ambientales (indicador aproximado de calidad ambiental) } \\
\text {, Delitos (indicador aproximado de seguridad pública) } \\
\text {, Teléfonos (infraestructura disponible de telecomunicaciones) } \\
\text {, Celulares (infraestructura disponible de telecomunicaciones) } \\
\text {, Internet (infraestructura disponible de telecomunicaciones) } \\
\text {, Parques industriales (infraestructura industrial disponible) } \\
\text {, Investigadores (capital humano para generar conocimiento e innovación) } \\
\text {, Centros de investigación (infraestructura para generación de conocimiento, } \\
\text { desarrollo tecnológico e innovación) }\end{array}$ \\
\hline Institucional & $\begin{array}{l}\text {, Capacidad financiera (nivel de autosuficiencia en las finanzas municipales) } \\
\text {, Dependencia financiera (nivel de subordinación a otros niveles de gobierno) } \\
\text {, Deuda pública (autosuficiencia y flexibilidad de las finanzas municipales) } \\
\text {, Ingreso per cápita (fortaleza y salud de las finanzas municipales) } \\
\text {, Inversión per cápita (capacidad de inversión pública del gobierno municipal) } \\
\text {, Reglamentos (calidad del marco reglamentario) } \\
\text {, Transparencia (mecanismos de transparencia) } \\
\text {, Catastro (calidad del catastro) } \\
\text {, Planeación (mecanismos y calidad de la planeación) } \\
\text {, Apertura de empresas (mejora regulatoria para la inversión) }\end{array}$ \\
\hline
\end{tabular}


Debe decirse que se llevó a cabo un primer cálculo incluyendo algunas variables que no se muestran en el cuadro anterior, sin embargo, no todas resultaron estadísticamente significativas para el modelo, por lo que suprimieron. Las que finalmente quedaron fueron las que se presentan en el cuadro 1 . Una vez obtenidas las variables definitivas, se realizaron cuatro cálculos por separado para determinar un índice por cada componente de competitividad. Finalmente, se realizó un promedio simple de los cuatro componentes para obtener el índice general. Los principales resultados se muestran a continuación.

\section{Resultados del índice de competitividad de ciudades mexicanas del CIDE}

Antes de comenzar con los resultados del índice, se considera importante presentar algunos datos que podrían ser útiles para entender la situación de las ciudades mexicanas. En el país existen 2.434 municipios. Para el año 2000, el país contaba con una población de $97 \mathrm{mi}$ llones 483 mil 412 habitantes. El 65\% vivía en zonas urbanas. De ese porcentaje, el 65\% se concentraba en sólo 9 ciudades. La ciudad de México está en primer lugar con 17 millones de habitantes. Le siguen Guadalajara, Monterrey y Puebla, cuya población rebasa los 2 millones de habitantes. Las restantes son Tijuana, León, Toluca, Ciudad Juárez y Torreón, que están por arriba del millón de habitantes. Todas estas ciudades son áreas metropolitanas (AM), a excepción de Ciudad Juárez 5 .

En cuanto a distribución de la población en el territorio, la zona central es la que tiene la mayor población, ya que en ella se encuentran las AM de Ciudad de México, Puebla, Toluca y León. Guadalajara se localiza en el Occidente y Monterrey en el Noreste. Torreón, Tijuana y Juárez están en el norte y las dos últimas son ciudades fronterizas con Estados Unidos.

Debido al volumen de población y a los niveles de producción, por la concentración de la actividad económica, el centro-norte del país se caracteriza por tener los mayores niveles, mientras que el sur y sureste tienen los niveles más bajos. También en el sur y sureste se encuentran los municipios con mayores índices de pobreza. En el cálculo del índice sólo se toman en cuenta municipios urbanos, sin embargo, los resultados muestran claras diferencias en ellos.

\section{a) Componente Económico}

Aquí se presenta a las ciudades con una mejor posición competitiva en cuanto a su estructura económica, perfil de desarrollo local y potencial de inserción en la economía global. Las variables más significativas para este componente fueron: la riqueza generada (producción bruta por habitante), el nivel salarial (sueldo promedio del personal ocupado), la estructura económica (índice de especialización local) y participación de la industria moderna en la economía ${ }^{6}$.

5 Datos de INEGI (2005) y CONAPO (2000).

6 La industria moderna se define como el porcentaje de producción bruta total de los subsectores papel, química, minerales no metálicos, metálica básica, productos metálicos y otras industrias manufactureras, con respecto a la producción total industrial. 
Los primeros lugares en el 2007 corresponden a Saltillo, Monclova, Monterrey, Puebla, San Luis Potosí, Querétaro, Ciudad Juárez, Aguascalientes, Toluca y Celaya. Todas estas se encuentran en la parte norte y centro de México y todas cuentan con una actividad primordialmente industrial. Saltillo, Monclova y Monterrey son las que tienen los valores más altos en producción. Saltillo y Monclova tienen una proporción mayor de participación de la industria moderna en la economía local, mientras que Monterrey destaca en cuanto a participación del comercio y servicios modernos. El resto de las ciudades mantienen niveles similares en todas las variables.

Monterrey, Puebla, Ciudad Juárez y Toluca son algunas de las ciudades más grandes del país en cuanto a población, con más de un millón de habitantes. Ciudad Juárez se encuentra en la frontera con Estados Unidos y tiene una estructura económica basada en la industria maquiladora, además de que su población está estrechamente ligada a la actividad del país vecino, ya sea por motivos de trabajo, educación o comercio.

En los resultados del 2003, Saltillo, Monclova y Monterrey también habían quedado en la primera, segunda y tercera posición de competitividad, por lo que mantuvieron su posición. Esta situación sólo se presentó en este componente, ya que en los tres restantes hubo cambios en la primera posición. De las primeras quince posiciones, las ciudades que mejoraron su posición competitiva fueron Puebla, Aguascalientes y Mexicali, mientras que aquellas que disminuyeron su posición pero todavía se encuentran entre las primeras quince fueron Querétaro, Toluca y Orizaba. San Luis Potosí conservó la quinta posición en ambos años. La Ciudad de México, Hermosillo, Guadalajara, Cuernavaca y Coatzacoalcos salieron de la lista de las quince primeras posiciones en el componente económico. Las que entraron a las primeras posiciones fueron Ciudad Juárez, Celaya, Matamoros, Chihuahua y Torreón. Las ciudades que se encuentran en las posiciones más bajas del índice corresponden a ciudades del sur y sureste del país.

Tomando en cuenta los resultados del índice en 2003 y 2007, los principales cambios en las primeras quince posiciones favorecieron a la región norte, ya que se sumaron tres ciudades y sólo salió una. La región central disminuyó su presencia de siete a seis, al salir una ciudad. Igualmente en el sur del país, que tenía dos ciudades y salió una. Entre los cambios más relevantes dentro de las variables se observa que la producción por habitante y el nivel salarial aumentaron sus niveles en las ciudades que mantuvieron su posición o que la mejoraron. La estructura económica local está cada vez más concentrada en los sectores comercio y servicios, aunque el industrial todavía sigue siendo relevante. La participación de la industria, comercio y servicios modernos, en general, mostraron disminución. Esto último afectó en mayor medida a las ciudades con las menores posiciones en el aspecto económico del índice.

\section{b) Componente Institucional}

Este componente se refiere a las condiciones ofrecidas por los gobiernos municipales para el desarrollo de las actividades económicas en las ciudades. Las primeras posiciones en el 2007 corresponden a Morelia, La Paz, Reynosa, Chihuahua, Durango, Mazatlán, Monterrey, Hermosillo, Tijuana y San Luis Potosí. Todas se encuentran en el centro y norte del país. Las 
características de los gobiernos municipales de estas ciudades en principio permitirían crear condiciones propicias a la competitividad.

Entre las variables más relevantes se encuentran la capacidad financiera, es decir la proporción de gasto corriente que se cubre con ingresos propios, los mecanismos de planeación, transparencia y el marco regulatorio. Particularmente, Morelia y Paz se encuentran dentro de las primeras posiciones en referencia a estos aspectos. El resto de las ciudades sobresale en alguno(s) de ellos. Por ejemplo, Reynosa destaca en cuanto a capacidad financiera y Chihuahua en capacidad financiera, transparencia y catastro.

Otras características importantes de las primeras posiciones, son que todas las ciudades se encuentran en el norte del país, a excepción de Morelia que está localizada en el centro. Tanto en Morelia, La Paz, Durango y Mazatlán, las actividades comerciales y de servicios son la base de su estructura económica. Mazatlán es una ciudad turística y portuaria. Tijuana colinda con Estados Unidos y predomina la industria maquiladora y el comercio. En Monterrey, Hermosillo, San Luis Potosí y Chihuahua hay más especialización industrial.

Todas las ciudades que en 2003 se encontraban en las primeras posiciones en este componente cambiaron su lugar. Esto es explicado en parte debido a que en esta última versión se incluyeron variables nuevas que no habían sido medidas anteriormente (en 2003 las variables que se incluían eran referentes al manejo de las finanzas públicas municipales). Pero, por otro lado, también demuestra que si bien las ciudades que resultaron más favorecidas en 2003 tienen fortalezas en cuanto al aspecto hacendario, de igual manera presentan debilidades en cuanto a mecanismos de transparencia, planeación y mejora regulatoria, marco reglamentario y catastro. Las ciudades que lograron mantenerse entre las primera quince fueron Chihuahua, Reynosa y Mazatlán, Los Cabos, Mexicali y Villahermosa, donde las tres primeras mejoraron su posición y las tres últimas la disminuyeron. Cabe destacar que en los resultados del 2003 las primeras posiciones estaban distribuidas entre ciudades del norte, centro y sur del país, mientras que para 2007, las del norte predominaron. Los últimos lugares de este componente corresponden a ciudades tanto del centro como el sur del país, las cuales muestran baja capacidad financiera, alta dependencia de otros niveles de gobierno y carencias en cuanto a marco reglamentario, mecanismos de planeación y transparencia.

\section{c) Componente Socio-demográfico}

La competitividad en este componente se refiere a las características poblacionales y a la estructura social que presentan las ciudades. Las primeras posiciones son ocupadas en 2007 por Chihuahua, Monterrey, Monclova, Ciudad Juárez, Saltillo, Tijuana, Hermosillo, Matamoros, Guadalajara y Querétaro. En estos diez primeros lugares se encuentran dos de las ciudades más grandes del país y la mayoría de ellas se sitúa en el norte de la república mexicana. Si bien en todas las ciudades de México se presentan desequilibrios sociales muy graves y fenómenos de marginación y exclusión social, estas ciudades, dadas sus características, serían las mejor posicionadas para mitigar parte de esa realidad de desigualdad, y con posibilidades de cuidar este perfil poblacional y social. 
Entre las variables más relevantes en este aspecto se encuentran el nivel de marginación, índice de desarrollo humano, estructura del empleo, nivel de pobreza y tasa de desocupación. En el caso de Chihuahua y Monterrey, ambas tienen los niveles más bajos de marginación, alto índice de desarrollo humano y baja proporción de población ocupada en el sector primario. En Tijuana y Ciudad Juárez, las dos fronterizas con Estados Unidos, hay alta proporción de población ocupada en el sector industrial, lo cual está relacionado con su actividad maquiladora. Querétaro es representativa en cuanto al índice de desarrollo humano.

A diferencia de los componentes económico e institucional, en éste Chihuahua se mantuvo en el primer lugar en las dos versiones del índice. Si se toman en cuenta las primeras quince posiciones en el índice, en ambos años hay mayor presencia de ciudades del norte del país, en 2007 aumentaron las del centro y no hubo presencia del sur. Una de las que entraron en 2007 fue la Ciudad de México, que es la más grande del país.

En general, todas las ciudades del estudio mostraron mejoras en cuanto a capacidad financiera, gasto de inversión por habitante e ingreso público por habitante. La dependencia financiera de otros niveles de gobierno disminuyó, lo cual puede estar relacionado con la menor distribución de fondos federales a través de participaciones. Sin embargo, la proporción de deuda pública aumentó. Los lugares más bajos del índice se encuentran entre ciudades del centro y sur del país, lugares donde predominan bajos niveles salariales, alta proporción de población ocupada en el sector primario y alta marginación.

\section{d) Componente Urbano-Ambiental}

El componente urbano-ambiental muestra la competitividad de las ciudades en cuanto a condiciones de infraestructura, servicios ofrecidos y equipamiento para el desarrollo de las actividades de las ciudades. Los primeros lugares en 2007 están ocupados por Monterrey, Ciudad de México, Toluca, San Luis Potosí, Culiacán, Puebla, Durango, Guadalajara, Querétaro y Aguascalientes. En este componente se encuentran ubicadas en los primeros diez lugares las tres áreas metropolitanas más grandes del país, mientras que las siete restantes corresponden a ciudades medias. Todas ellas están ubicadas en el norte y centro del país. Si bien existe un déficit importante en materia de infraestructura urbana en todas las ciudades del país, en principio es en éstas en las que se ofrece un nivel de infraestructura interesante comparativamente y en proporción a su tamaño y amplitud de actividades.

Entre las variables más significativas se pueden mencionar la disponibilidad de infraestructura en telecomunicaciones, la jerarquía urbana, la infraestructura y capital humano para la generación de conocimiento. En estos aspectos, es claro el predominio de las ciudades más grandes del país, entre las que se encuentran Ciudad de México, Monterrey, Toluca, Puebla y Guadalajara. La mayor disponibilidad de infraestructura y personal para la generación de conocimiento se encuentra, por un amplio margen, concentrada en la Ciudad de México. La mayoría de las ciudades dentro de los primeros diez lugares tienen una estructura económica basada en la actividad industrial, a excepción de Culiacán, que está basada en el comercio y servicios, y Durango, basada en el comercio. 
Las quince primeras posiciones cambiaron con respecto al 2003. Sin embargo, resulta interesante mencionar que de las primeras 15 ciudades del 2003, 12 continuaron en la lista. Entre las que mejoraron su posición se encuentran Monterrey, San Luis Potosí, Ciudad de México y Puebla, mientras que Toluca, Culiacán, Querétaro y Tijuana perdieron posiciones. Las que salieron de la lista fueron Cancún y Mérida, ciudades turísticas del sur del país, y las que entraron fueron Cuernavaca y Chihuahua. En este componente urbano también se aumentaron variables para el cálculo de 2007, no obstante, los cambios en las posiciones fueron menos perceptibles que en el caso del componente institucional. Las ciudades con las menores posiciones dentro del índice se encuentran tanto en el sur como en el norte del país y presentan bajos niveles de infraestructura para telecomunicaciones y generación de conocimiento, así como bajas proporciones de personal para la investigación científica.

\section{e) Promedio de componentes}

El promedio de los cuatro componentes de competitividad del estudio considera de manera integrada las características económicas, institucionales, socio-demográficas y urbano-ambientales. El primer lugar en 2007 lo ocupa Monterrey, el segundo Chihuahua y el tercero la Ciudad de México. Las siguientes ciudades son San Luis Potosí, Ciudad Juárez, Tijuana, Aguascalientes, Hermosillo, Saltillo y Toluca. En estos primeros diez lugares se encuentran dos de la tres áreas metropolitanas más grandes del país, aunque Guadalajara, que es la tercera en importancia, se encuentra en el lugar once del conjunto de ciudades. El resto de las ciudades corresponden a ciudades medias, tanto del norte del país como de la zona central. Si bien las ciudades mexicanas no están bien posicionadas en términos de competitividad si las comparamos con las ciudades más competitivas del mundo, como Tokio, Londres, París, Nueva York, Milán o Munich, es claro que las once ciudades mencionadas ofrecen, con sus limitantes, las mejores condiciones y el mejor ambiente para la competitividad en México7.

Querétaro, que en 2003 había obtenido la primera posición, bajó al lugar 12 en 2007, su lugar fue ocupado por Monterrey, que en 2003 se encontraba en segundo lugar. De las ciudades que ocuparon los primeros 15 lugares de competitividad urbana en 2003, 12 se conservaron en la lista y sólo tres salieron. Entre las que mejoraron su posición se encuentran Monterrey, Chihuahua, Ciudad de México, Tijuana y Ciudad Juárez. Guadalajara se mantuvo en la posición 11 en ambos años. Mientras que Saltillo, Mexicali y Toluca perdieron posiciones, aunque se conservan dentro de la lista. Las que salieron fueron Cancún, Tampico y Los Cabos, y las que entraron en 2007 fueron Hermosillo, Durango y Reynosa.

En los cuatro componentes, las ciudades que predominan en los primeros lugares son las del norte y centro del país, mientras que las del sur parecen estar más relegadas. De acuerdo a los resultados del índice, las ciudades más competitivas en términos económicos son aquellas cuya estructura económica está basada en el sector industrial, donde las fronterizas, como Ciudad Juárez y Tijuana, destacan por su actividad maquiladora. Mientras tanto, ciudades turísticas como Cancún y Los Cabos, que en 2003 habían ocupado mejores posiciones, disminuyeron su competitividad para 2007.

7 Las ciudades mencionadas son, según Van Winden (2006), las de mayor competitividad a nivel mundial. 
En las siguientes gráficas se pueden observar estos cambios con mayor claridad, ya que muestran la comparación de los cuatro componentes de acuerdo al tipo de ciudades, por lo tanto, permiten identificar los diversos perfiles de competitividad en México. En la figura 1 se presenta la comparación entre Monterrey y la Ciudad de México. Las dos ciudades muestran un perfil de competitividad amplio, es decir, que abarca más de cada componente. Monterrey fue la que obtuvo la primera posición en el índice de competitividad promedio, así como en el componente urbano. Como ya se había comentado anteriormente, es una ciudad predominantemente industrial y con la mayor proporción de disponibilidad de infraestructura para las telecomunicaciones. La Ciudad de México tiene una menor proporción en los componentes que Monterrey, sin embargo, su perfil es parecido. En ambas destaca más el aspecto urbano.

\section{FIGURA 1. PERFILES DE COMPETITIVIDAD EN DOS CIUDADES GRANDES: MONTERREY Y CIUDAD DE MÉXICO, 2007}

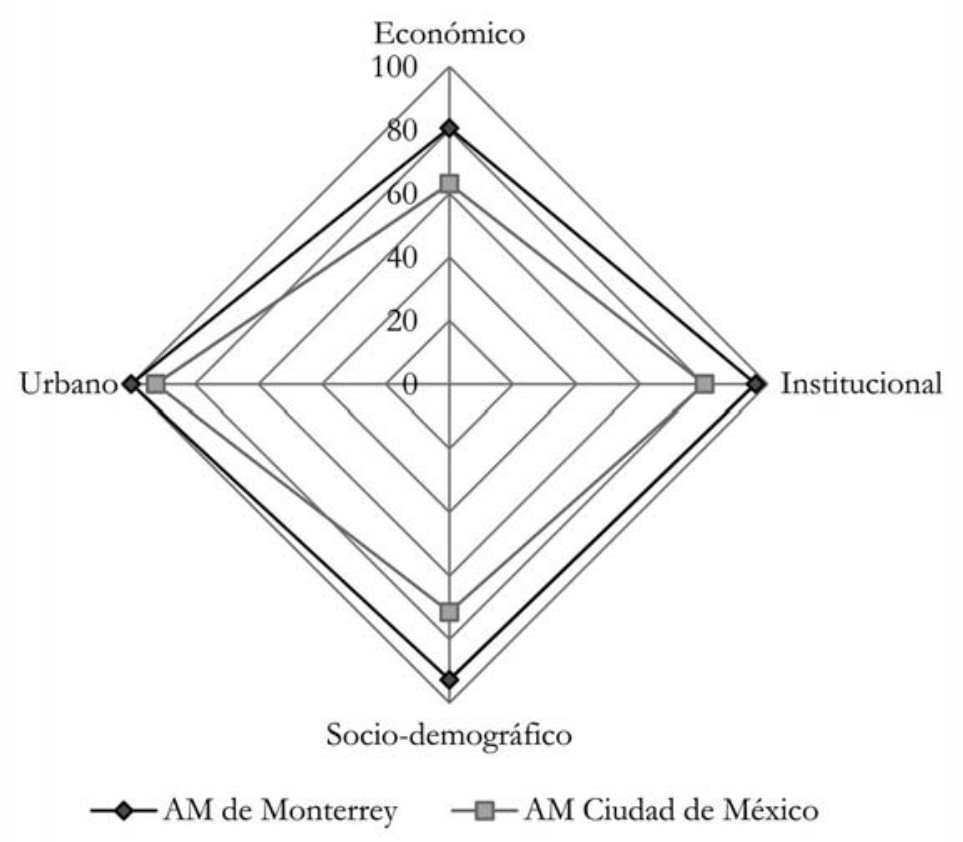

Fuente: Elaboración propia

La figura 2 presenta a dos ciudades fronterizas. A diferencia de la gráfica anterior, este perfil está más orientado al aspecto institucional y socio-demográfico, y son más bajos en el económico y en el urbano. En estas dos ciudades, aunque destacaron por su actividad industrial, no necesariamente cuentan con las mejores condiciones de infraestructura y equipamiento que caracterizan a otras ciudades, sin embargo, cuentan con otras ventajas como la proporción de producción por habitante. 
FIGURA 2. PERFILES DE COMPETITIVIDAD EN DOS CIUDADES FRONTERIZAS: CIUDAD JUÁREZ Y TIJUANA, 2007

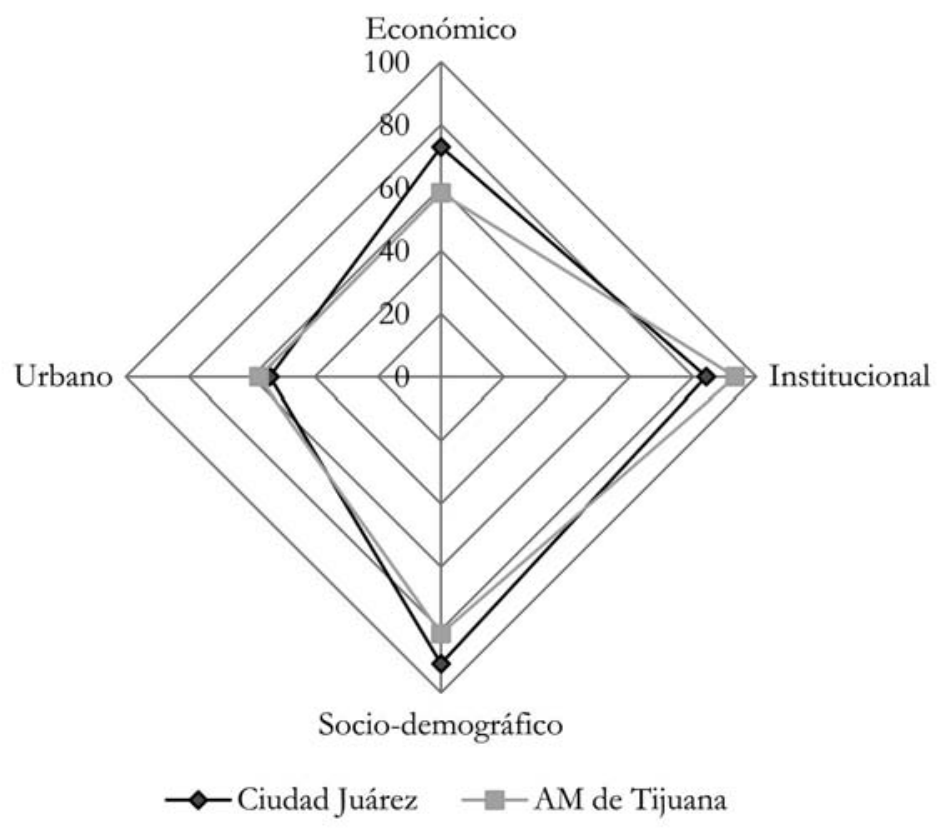

Fuente: Elaboración propia

La figura 3 muestra dos ciudades turísticas, una en el sur del país, Cancún, y otra en el norte, Los Cabos. En este tipo de ciudades el perfil de competitividad es más reducido que en los dos casos anteriores, es decir, que los niveles alcanzados en cada uno de los componentes es menor. Aun así, se puede observar que la competitividad tiende a ser menor en los componentes económicos y urbanos y mayor en el socio-demográfico e institucional. En el índice de 2003 estas dos ciudades se situaron entre las primeras quince con mejores condiciones ofrecidas en relación al manejo de las finanzas públicas municipales. Para el índice de 2007, y debido a las nuevas variables introducidas, sus posiciones cambiaron. Los Cabos obtuvo un menor valor en el componente pero siguió entre los primeros lugares. En cambio Cancún salió de esta parte de la lista. Es importante mencionar también que en estas ciudades la actividad turística redunda en bajos niveles de desocupación, sin embargo, las condiciones que ofrecen en cuanto a infraestructura y servicios están concentradas en las zonas hoteleras y no siempre favorecen a las zonas de la población residente. 
FIGURA 3. PERFILES DE COMPETITIVIDAD EN DOS CIUDADES TURÍSTICAS:

CANCÚN Y LOS CABOS, 2007

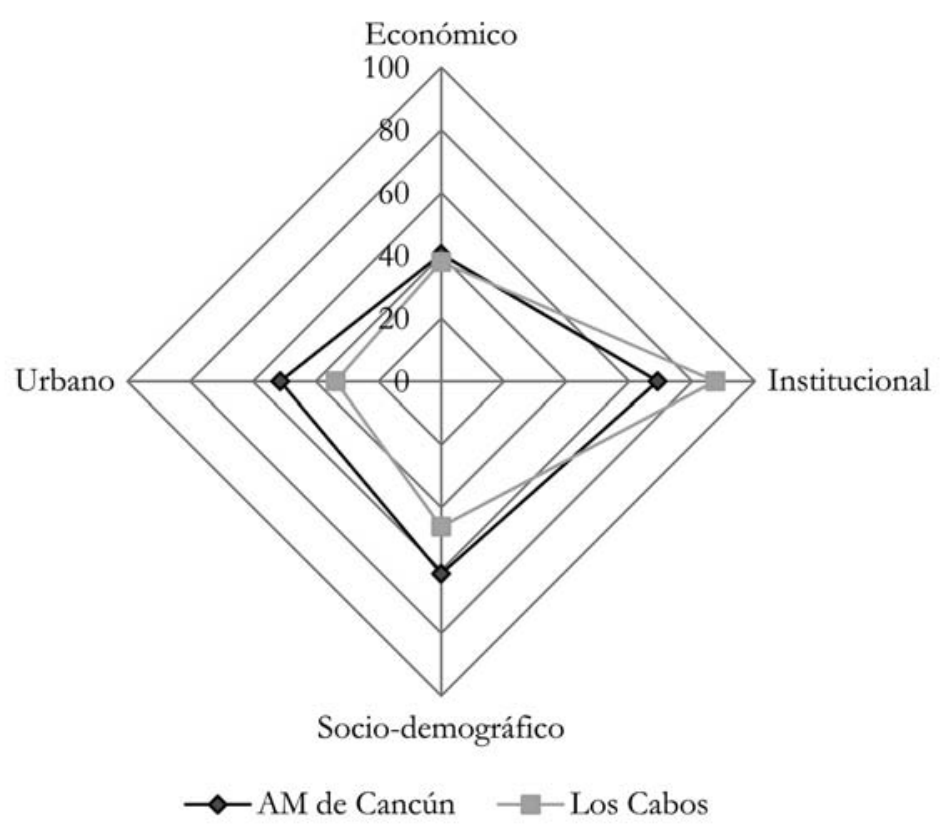

Fuente: Elaboración propia

Si bien el cálculo del índice de competitividad de ciudades mexicanas está supeditado a la existencia de datos publicados a nivel municipal o zona metropolitana, los resultados hasta ahora presentados muestran algunas tendencias en la estructura de competitividad de las ciudades del país a partir de 2003, en las cuales la pérdida o ganancia de posiciones están influidas por elementos diversos (desde desastres naturales, cuestiones económicas locales, políticas, laborales y de visión de los gobiernos municipales) que no están cuantificados en el índice, pero que se ven reflejados en las posiciones obtenidas en cada una de las ciudades.

\section{Comentarios finales}

Es claro que las ciudades deben atender la agenda de promoción de la competitividad en el mundo contemporáneo. El contexto internacional muestra la importancia de que cada país se dote de un conjunto de "motores para la competitividad" construidos desde el ámbito urbano, en el cual las ciudades crean los ambientes propicios para la competitividad y facilitan la conexión de los diversos componentes de la misma.

Por ello, se requiere de métodos de observación de los avances y dificultades que las ciudades encuentran en ese proceso, y esto ha dado lugar al diseño de diversos índices de medición de la competitividad para el ámbito de las ciudades. Si bien el llevar a cabo ejercicios de medición 
de competitividad de ciudades es recomendable, y en México ya existen diversos ejercicios para ello -como el que se presenta en este documento-; se trata tan solo de aproximaciones para observar una realidad compleja. Es necesario, por tanto, ser cautelosos en el uso de estos instrumentos de medición, pues se requiere complementarlos con datos cualitativos, establecer un diálogo con los actores empresariales, gubernamentales y sociales de las ciudades, antes de formarse una opinión de la situación que presentan.

Cada ciudad despliega esfuerzos, estrategias, acuerdos y una acción pública para la competitividad que no se ve reflejada necesariamente en los índices. Cada ciudad, por tanto, debe tomar el índice como una referencia comparativa y de su evolución en el tiempo, pero tan solo aproximada a su realidad compleja, por lo que deberá llevar a cabo un diagnóstico más amplio y detallado. Dicho diagnóstico deberá dar lugar a un diálogo entre los diversos grupos y actores locales, al establecimiento de acuerdos para la cooperación y la confección de políticas urbanas para la competitividad. Es este tipo de dinámica de construcción de redes locales lo que puede tener un impacto importante, al menos un impacto más importante que el solo hecho de revisar un índice cuantitativo, que si bien es útil como referencia estática, es débil como diagnóstico de situaciones dinámicas particulares.

Cabe mencionar, para terminar, que en el caso mexicano hay una agenda urgente de reformas y esfuerzos de fortalecimiento hacia los gobiernos municipales. En el momento actual, este nivel de gobierno no está suficientemente equipado para atender con eficacia la agenda de competitividad urbana. Es necesario reflexionar sobre los periodos de gobierno municipal, sobre el diseño institucional que todavía prevalece en el Cabildo, y sobre la debilidad de los instrumentos e instancias de coordinación metropolitana. Todos estos aspectos presentan debilidades estructurales de los gobiernos municipales si se comparan con otros gobiernos urbanos en el mundo, es decir, con los gobiernos de ciudades con los cuales deben competir las ciudades mexicanas. Igualmente puede hacerse referencia a la falta de sistemas de planeación de largo plazo, a la débil profesionalización de los funcionarios locales, y a los todavía poco frecuentes esquemas de cooperación local para la competitividad. Si bien es necesario resaltar que existen hoy en día muchas experiencias de gestión municipal innovadoras y atentas a la competitividad, es necesario aceptar que todavía el trecho por recorrer es largo, y que el resto de los niveles de gobierno deben llevar a cabo un esfuerzo más decidido por fortalecer las capacidades competitivas de las ciudades mexicanas. El proyecto de competitividad de ciudades conducido por el CIDE se plantea avanzar en estos aspectos en el futuro inmediato.

El cálculo de este índice de competitividad es sólo un primer paso en la medición de la competitividad urbana en el país, ya que en un futuro próximo se realizarán diversos ejercicios para incluir también variables de tipo cualitativo que no pueden obtenerse de publicaciones estadísticas, pero que pueden conseguirse a través de encuestas y foros de discusión. Sin duda, al conjuntar tanto elementos cuantitativos como cualitativos, la medición de la competitividad urbana logrará apegarse cada vez más a la realidad y servir entonces como un instrumento para el diseño y formulación de políticas que redunden en un mejor desempeño, tanto de los gobiernos como de las economías locales en beneficio de su población residente. 


\section{Referencias bibliográficas}

Begg, I. (1999). Cities and Competitiveness. Urban Studies, vol. 36, núm. 5/6, mayo, pp. $795-810$.

Begg, I. (2002). Urban Competitiveness. Policies for dynamic cities. Gran Bretaña: The Policy Press.

Berg, L. \& Braun, E. (1999). Urban competitiveness, marketing and the need for organising capacity. Urban Studies, 36 (5-6), pp. 987-999.

Bouinot, J. (2002). La Ville Compétitive. Les clefs de la nouvelle gestion urbaine. París: Ed. Economica.

Cabrero, E. (2002). Innovación en gobiernos locales. México: Centro de Investigación y Docencia Económicas.

Cabrero, E. (2005). Acción pública y desarrollo local. México: Fondo de Cultura Económica.

Cabrero, E., Orihuela, I. \& Ziccardi, A. (2003). Ciudades competitivas - ciudades cooperativas: conceptos clave y construcción de un índice para ciudades mexicanas, en Arce, Cabrero y Ziccardi (Eds.). Ciudades del siglo XXI: ¿competitividad o cooperación? México: Miguel Angel Porrúa.

CONAPO (2000). Delimitación de zonas metropolitanas de México. México.

Giannias, D. (1998). A Quality of Life Based Ranking of Canadian Cities. Urban Studies, vol. 35, n¹2, pp.2241-2251.

Graham, S. (1999). Global grids of glass: on global cities, telecommunications and planetary urban networks. Urban Studies, vol. 36 (5-6), 929-949.

Gordon, I. (1999). Internationalisation and Urban Competition. Urban Studies, vol. 36, núm. 5/6, pp. 1001-1016.

Kresl, P. (1995). The determinants of urban competitiveness, en P. Kresl y G. Gappert (Eds.). North American cities and the global economy: challenges and opportunities. Londres: Sage Publications.

Kresl, P. \& Balwant S. (1999). Competitiveness and the urban economy: twenty-four large US metropolitan areas. Urban Studies, vol. 36 (5-6), 1017-1027.

INEGI (2005). Finanzas públicas estatales y municipales de México. México.

Lever, W.F. \& Turok I. (1999). Competitive Cities: Introduction to the Review. Urban Studies, vol. 36, núm. 5/6, mayo, pp. 791-794.

Malecki, E. (2002). Hard and soft networks for urban competitiveness. Urban Studies, vol. 39 (5-6), 929-945.

McCauley, S. (2003). Methodology and principles of analysis. IMD World Competitiveness Yearbook.

Moori-Koenig, V. \& Yoguel, G. (1998). El desarrollo de capacidades innovativas de las firmas en un medio de escaso desarrollo del sistema local de innovación. Instituto de Industrias, UNGS, Documento de Trabajo, 9, San Miguel.

Musso, E. y Castagnino, P. (1997). Avantages compétitives des villes -une approche comparative dans le cadre de l'Europe du Sud. Revue d'Economie Régionale et Urbaine, $\mathrm{n}^{\circ} 1$, pp.67-96.

OCDE (2006). Competitive Cities in the Global Economy. OCDE.

Pastor, M. (2006). Cohesion and Competitiveness: Business Leadership for Regional Growth and Social Equity. Competitive Cities in the Global Economy. OCDE. 
Porter, M. (1991). La ventaja competitiva de las naciones. España: Plaza \& Janes Editores.

Porter, M. (1996). Competitive Advantage, Agglomeration Economics, and Regional Policy. International Regional Science Review, vol. 19, no. 1 \& 2, pp. 85-93, 1996.

Porter, M. (1995). The competitive advantage of the inner city. Harvard Business Review, vol. 73 no. 3, mayo-junio, pp. 55-71.

Raco, M. (1999). Competition, collaboration and the new industrial districts: examining the institutional turn in local economic development. Urban Studies, vol. 36 (5-6), 951-968.

Rogerson, R. (1999). Quality of life and city competitiveness. Urban Studies, vol. 36 (5-6), 969-985.

Sobrino, J. (2002). Competitividad y ventajas competitivas: revisión teórica y ejercicio de aplicación a 30 ciudades de México. Estudios Demográficos y Urbanos, vol. 17, núm. 2.

Sobrino, J. (2003) Competitividad de la ciudades en México. México: El Colegio de México.

Van Winden, W. (2006). Globalisation and Urban Competitiveness: Challenges for Different Types of Urban Regions. Competitive Cities in the Global Economy. OCDE. 
